\title{
Highly $\pi$-Extended TTF Analogues with a Conjugated Macrocyclic Enyne Core
}

\author{
Guang Chen, Li Wang, David W. Thompson, Yuming Zhao* \\ Department of Chemistry, Memorial University of Newfoundland \\ St. John's, Newfoundland A1B 3X7, Canada \\ Email: yuming@mun.ca
}

\section{Table of Contents}

1. Synthetic Details

S 1

2. NMR Spectra of Compounds $\mathbf{4}-\mathbf{8}$

S 8

\section{Synthetic Details}

\subsection{General}

Chemicals were purchased from commercial suppliers and used directly without purification. $\mathrm{CH}_{2} \mathrm{Cl}_{2}$ was distilled from sodium/benzophenone before its use in the Corey-Fuchs reactions for $\mathbf{5 a}-\mathbf{b}$. $\mathrm{Et}_{3} \mathrm{~N}$ was distilled from $\mathrm{LiH}$ prior to use in Sonogashira coupling. All reactions were conducted in standard, dry glassware and under an inert atmosphere of nitrogen unless otherwise noted. Evaporation and concentration were carried out with a water-aspirator. Flash column chromatography was performed using 240-400 mesh silica gel obtained from VWR International. Thin-layer chromatography (TLC) was carried out with silica gel 60 F254 covered on plastic sheets and visualized by UV light or $\mathrm{KMnO}_{4}$ stain. Melting points (m.p.) were measured with a Fisher-Jones melting point apparatus and are uncorrected. ${ }^{1} \mathrm{H}$ and ${ }^{13} \mathrm{C}$ NMR spectra were measured on the Bruker Avance $500 \mathrm{MHz}$ spectrometer. Chemical shifts are reported in ppm downfield from the signal of the internal reference $\mathrm{SiMe}_{4}$. Coupling constants $(J)$ are given in Hz. Infrared spectra (IR) were recorded on a Bruker Tensor 27 spectrometer. APCI mass spectra (MS) were measured on an Agilent 1100 series LCMSD spectrometer, and MALDI-TOF MS were measured on an Applied Biosystems Voyager instrument using dithranol as the matrix. Compounds $\mathbf{2} \mathbf{a}$ and $\mathbf{2} \mathbf{b}$ were prepared using the methods reported in the literature. ${ }^{1}$

\footnotetext{
${ }^{1}$ (a) Moore, A. J.; Bryce, M. R. Tetrahedron Lett. 1992, 33, 1373. (b) Chen, G.; Zhao, Y. Tetrahedron Lett. 2006, 47, 5069 .
} 


\subsection{Synthetic Details for Compounds 4-8}

Synthesis of 10-(4,5-bis(methylthio)-1,3-dithiol-2-ylidene)anthracen-9(10H)-one $(4 a) .^{2}$

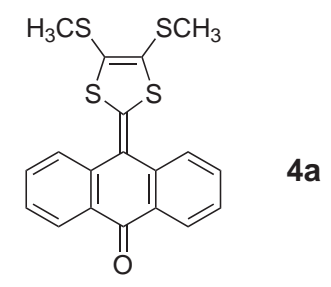

$\mathrm{P}(\mathrm{OEt})_{3}(20 \mathrm{~mL})$ was thoroughly degassed and heated to $155^{\circ} \mathrm{C}$ in a $50 \mathrm{~mL}$ round-bottom flask. Anthraquinone (3) (1.2 g, $5.8 \mathrm{mmol}$ ) was then charged, followed by slow addition of a solution of thione $\mathbf{2 a}(1.2 \mathrm{~g}, 5.3 \mathrm{mmol})$ in xylenes $(10 \mathrm{~mL})$ over a period of $40 \mathrm{~min}$. During the addition of thione $\mathbf{2 a}$ at $c a .20 \mathrm{~min}$, a second portion of anthraquinone (3) (0.80 g, $3.8 \mathrm{mmol}$ ) was added to the reaction. After addition of thione was complete, the reaction mixture was stirred for $1 \mathrm{~h}$ and then concentrated in vacuo at $95^{\circ} \mathrm{C}$ to ca. $6 \mathrm{~mL}$. The residue was chromatographied with gradient elution $\left(30 \% \mathrm{CH}_{2} \mathrm{Cl}_{2}\right.$ in hexanes, and then $80 \% \mathrm{CH}_{2} \mathrm{Cl}_{2}$ in hexanes) to afford product $\mathbf{4 a}$ as a red solid $(0.32 \mathrm{~g}, 0.83 \mathrm{mmol}, 16 \%)$. m.p. $209-210{ }^{\circ} \mathrm{C}$; ${ }^{1} \mathrm{H}$ NMR $\left(500 \mathrm{MHz}, \mathrm{CDCl}_{3}\right): \delta 8.27(\mathrm{dd}, J=8.5,1.0 \mathrm{~Hz}, 2 \mathrm{H}), 7.78(\mathrm{~d}, J=8.0 \mathrm{~Hz}, 2 \mathrm{H}), 7.65$ $(\mathrm{td}, J=7.5,1.0 \mathrm{~Hz}, 2 \mathrm{H}), 7.45(\mathrm{t}, J=7.5 \mathrm{~Hz}, 2 \mathrm{H}), 2.42\left(\mathrm{~s}, 6 \mathrm{H}, \mathrm{SC}_{3}\right) ;{ }^{13} \mathrm{C} \mathrm{NMR}(125 \mathrm{MHz}$, $\mathrm{CDCl} 3): \delta 183.5(\underline{\mathrm{C}}=\mathrm{O}), 140.1,138.6,131.9,130.7,127.3,127.0,126.9,126.2,119.4,19.3$ $\left(\mathrm{SCH}_{3}\right)$; FTIR (neat) 3060, 2918, 1647, 1593, $1484 \mathrm{~cm}^{-1}$; APCI-MS m/z calcd for $\mathrm{C}_{19} \mathrm{H}_{14} \mathrm{OS}_{4}$ 386.0 , found $387.0\left(100,[\mathrm{M}+\mathrm{H}]^{+}\right)$.

Synthesis of 10-(4,5-bis(decylthio)-1,3-dithiol-2-ylidene)anthracen-9(10H)-one (4b).

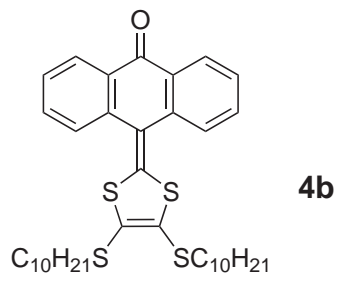

Compound $\mathbf{4 b}$ was synthesized by reacting thione $\mathbf{2 b}(3.0 \mathrm{~g}, 6.3 \mathrm{mmol})$ with anthraquinone $(2.0 \mathrm{~g}, 9.6 \mathrm{mmol})$ in $\mathrm{P}(\mathrm{OEt})_{3}(25 \mathrm{~mL})$ and xylene $(25 \mathrm{~mL})$ using the similar procedures as described in the synthesis of $\mathbf{4 a}$. Pure $\mathbf{4 b}(2.0 \mathrm{~g}, 3.1 \mathrm{mmol}, 49 \%)$ was obtained after gradient column chromatography as a red solid. m.p. $48-50{ }^{\circ} \mathrm{C} ;{ }^{1} \mathrm{H}$ NMR $\left(500 \mathrm{MHz}, \mathrm{CDCl}_{3}\right): \delta 8.27$

\footnotetext{
${ }^{2}$ The synthesis of compound 4a was previously reported in: Batsanov, A. S.; Bryce, M. R.; Coffin, M. A.; Green, A.; Hester, R. E.; Howard, J. A. K.; Lednev, I. K.; Martín, N.; Moore, A. J.; Moore, J. N.; Ortí, E.; Sánchez, L.; Savirón, M.; Viruela, P. M.; Viruela, R.; Ye, T.-Q. Chem. Eur. J. 1998, 4, 2580.
} 
$(\mathrm{dd}, J=7.5,1.0 \mathrm{~Hz}, 2 \mathrm{H}), 7.79(\mathrm{~d}, J=8.0 \mathrm{~Hz}, 2 \mathrm{H}), 7.65(\mathrm{td}, J=8.0,1.0 \mathrm{~Hz}, 2 \mathrm{H}), 7.44(\mathrm{t}$, $J=7.5 \mathrm{~Hz}, 2 \mathrm{H}), 2.80(\mathrm{t}, J=7.0 \mathrm{~Hz}, 4 \mathrm{H}), 1.63-1.57(\mathrm{~m}, 4 \mathrm{H}), 1.39-1.35(\mathrm{~m}, 4 \mathrm{H}), 1.30-1.24$ $(\mathrm{m}, 24 \mathrm{H}), 0.87(\mathrm{t}, J=7.0 \mathrm{~Hz}, 6 \mathrm{H}) ;{ }^{13} \mathrm{C}$ NMR $\left(125 \mathrm{MHz}, \mathrm{CDCl}_{3}\right): \delta 183.5(\underline{\mathrm{C}}=\mathrm{O}), 140.6$, 138.8, 131.8, 130.7, 127.4, 127.3, 126.7, 126.2, 118.8, 36.5, 31.9, 29.7, 29.53, 29.50, 29.3, 29.1, 28.5, 22.7, 14.1; FTIR (neat) 3060, 2924, 2853, 1658, 1596, 1485, $1466 \mathrm{~cm}^{-1}$; APCI-MS m/z calcd for $\mathrm{C}_{37} \mathrm{H}_{50} \mathrm{OS}_{4} 638.3$, found $639.2\left(100,[\mathrm{M}+\mathrm{H}]^{+}\right)$.

\section{Synthesis of 4,5-bis(methylthio)-2-(10-(dibromomethylene)anthracen-9(10H)-yli- dene)-1,3-dithiole (5a).}

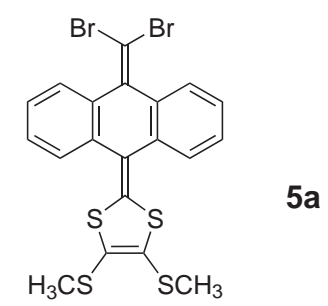

Compound 4a (282 $\mathrm{mg}, 0.731 \mathrm{mmol}), \mathrm{CBr}_{4}(987 \mathrm{mg}, 2.98 \mathrm{mmol})$ were mixed in a 100 $\mathrm{mL}$ oven-dried round-bottom flask. The mixture was flame-dried under vacuum for $5 \mathrm{~min}$, then dry $\mathrm{CH}_{2} \mathrm{Cl}_{2}(20 \mathrm{~mL})$ was added. A solution of $\mathrm{PPh}_{3}(1.60 \mathrm{~g}, 6.11 \mathrm{mmol})$ in $\mathrm{CH}_{2} \mathrm{Cl}_{2}$ was added, and the mixture was stirred overnight, and then concentrated in vacuo. The residue was subjected to silica flash column chromatography using hexanes as the eluent to afford $\mathbf{5 a}$ (248 mg, $0.458 \mathrm{mmol}, 63 \%$ ) as an orange crystal. m.p. $140{ }^{\circ} \mathrm{C}$ (dec.); ${ }^{1} \mathrm{H} \mathrm{NMR} \mathrm{(500} \mathrm{MHz,}$ $\left.\mathrm{CDCl}_{3}\right): \delta 7.87(\mathrm{~d}, J=8.0 \mathrm{~Hz}, 2 \mathrm{H}), 7.60(\mathrm{~d}, J=7.5 \mathrm{~Hz}, 2 \mathrm{H}), 7.32(\mathrm{t}, J=7.5,2 \mathrm{H}), 7.25(\mathrm{t}, J$ $=7.5 \mathrm{~Hz}, 2 \mathrm{H}), 2.42\left(\mathrm{~s}, 6 \mathrm{H}, \mathrm{SC}_{3}\right) ;{ }^{13} \mathrm{C} \mathrm{NMR}\left(125 \mathrm{MHz}, \mathrm{CDCl}_{3}\right): \delta 139.6,136.0,133.8,132.4$, 127.9, 127.4, 125.95, 125.85, 125.0, 122.2, 89.8 ( $\left.\underline{\mathrm{CBr}}_{2}\right)$, 19.1 ( $\left.\underline{\mathrm{SCH}}_{3}\right)$; FTIR (neat) 3059, 2919, 1682, 1534, 1499, $1448 \mathrm{~cm}^{-1}$; APCI-MS $m / z$ calcd for $\mathrm{C}_{20} \mathrm{H}_{14}{ }^{79} \mathrm{Br}_{2} \mathrm{~S}_{4} 539.8$, found 539.8 (34, $\left.[\mathrm{M}]^{+}\right)$.

Synthesis of 4,5-bis(decylthio)-2-(10-(dibromomethylene)anthracen-9(10H)-ylidene)-1,3-dithiole (5b).

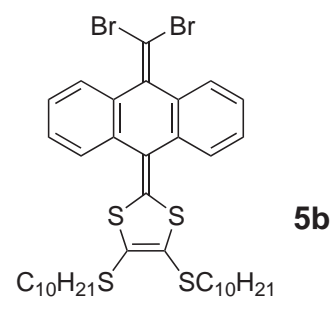

Compound $\mathbf{5 b}$ was prepared using the similar procedures as described in the synthesis of 5a: $4 \mathbf{b}(1.21 \mathrm{~g}, 1.89 \mathrm{mmol}), \mathrm{CBr}_{4}(2.7 \mathrm{~g}, 8.2 \mathrm{mmol}) \mathrm{PPh}_{3}(4.2 \mathrm{~g}, 16 \mathrm{mmol})$ in $\mathrm{CH}_{2} \mathrm{Cl}_{2}$ 
(30 mL). Pure 5b (1.36 g, $1.71 \mathrm{mmol}, 91 \%)$ was obtained through column chromatography using $10 \% \mathrm{CH}_{2} \mathrm{Cl}_{2}$ in hexanes as the eluent as a yellow oil . ${ }^{1} \mathrm{H} \mathrm{NMR}\left(500 \mathrm{MHz}, \mathrm{CDCl}_{3}\right.$ ): $\delta 7.87(\mathrm{dd}, J=7.5,1.0 \mathrm{~Hz}, 2 \mathrm{H}), 7.63(\mathrm{~d}, J=7.5 \mathrm{~Hz}, 2 \mathrm{H}), 7.31(\mathrm{td}, J=7.5,1.0 \mathrm{~Hz}, 2 \mathrm{H})$, 7.25-7.22 (m, 2H), 2.88-2.77 (m, 4H), 1.67-1.61 (m, 4H), 1.43-1.39 (m, 4H), 1.30-1.20 (m, $24 \mathrm{H}), 0.87(\mathrm{t}, J=7.0 \mathrm{~Hz}, 6 \mathrm{H}) ;{ }^{13} \mathrm{C}$ NMR $\left(125 \mathrm{MHz}, \mathrm{CDCl}_{3}\right): \delta 139.7,136.1,133.8,132.9$, 127.9, 127.4, 126.4, 125.7, 124.9, 121.3, $89.6\left(\mathrm{CBr}_{2}\right), 36.3,31.9,29.7,29.6,29.5,29.3,29.1$, 28.6, 22.7, 14.1; FTIR (neat) 3061, 2924, 2852, 1951, 1538, 1496, $1449 \mathrm{~cm}^{-1}$; APCI-MS m/z calcd for $\mathrm{C}_{38} \mathrm{H}_{50}{ }^{79} \mathrm{Br}_{2} \mathrm{~S}_{4}$ 794.1, found $794.2\left(38,[\mathrm{M}]^{+}\right)$.

\section{Synthesis of (3-(10-(4,5-bis(methylthio)-1,3-dithiol-2-ylidene)anthracen-9(10H)- ylidene)penta-1,4-diyne-1,5-diyl)bis(trimethylsilane) (6a).}

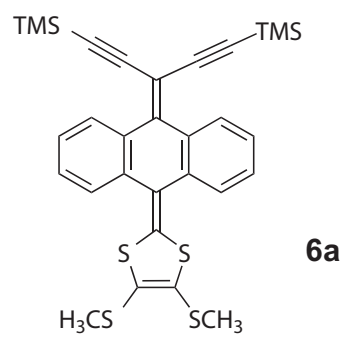

Compound 5a (210 mg, $0.387 \mathrm{mmol}), \mathrm{Pd}\left(\mathrm{PPh}_{3}\right)_{4}(45 \mathrm{mg}, 0.039 \mathrm{mmol}), \mathrm{CuI}(22 \mathrm{mg}, 0.116$ mmol) were charged into a $100 \mathrm{~mL}$ round-bottom flask. $\mathrm{Et}_{3} \mathrm{~N}(100 \mathrm{~mL})$ was added and the mixture was rigorously degassed by three freeze-pump-thaw cycles. Trimethylsilyl acetylene $(6.0 \mathrm{~mL}, 43 \mathrm{mmol})$ was added, and the mixture was heated to $60{ }^{\circ} \mathrm{C}$ under stirring for 2 days. When the reaction was complete as checked by TLC analysis, the reaction content was diluted with $\mathrm{CH}_{2} \mathrm{Cl}_{2}$, washed with aq. $\mathrm{NH}_{4} \mathrm{Cl}$ and $\mathrm{H}_{2} \mathrm{O}$. The organic layer was dried over $\mathrm{MgSO}_{4}$, followed by vacuum evaporation and column chromatography using $5 \% \mathrm{CH}_{2} \mathrm{Cl}_{2}$ in hexanes. Pure 6a (105 mg, $0.182 \mathrm{mmol}, 47 \%)$ was afforded as a red-brown solid. m.p. 165$167{ }^{\circ} \mathrm{C} ;{ }^{1} \mathrm{H} \operatorname{NMR}\left(500 \mathrm{MHz}, \mathrm{CDCl}_{3}\right): \delta 8.27(\mathrm{~d}, J=7.5 \mathrm{~Hz}, 2 \mathrm{H}), 7.59(\mathrm{~d}, J=7.5 \mathrm{~Hz}, 2 \mathrm{H})$, $7.34(\mathrm{t}, J=7.5,2 \mathrm{H}), 7.22(\mathrm{t}, J=7.5 \mathrm{~Hz}, 2 \mathrm{H}), 2.41\left(\mathrm{~s}, 6 \mathrm{H}, \mathrm{SC}_{3}\right), 0.21\left(\mathrm{~s}, 18 \mathrm{H}, \mathrm{Si}\left(\mathrm{C}_{3}\right)_{3}\right)$; ${ }^{13} \mathrm{C}$ NMR $\left(125 \mathrm{MHz}, \mathrm{CDCl}_{3}\right): \delta 148.0,135.5,132.6,132.5,127.8,127.7,126.2,125.5,124.9$, 123.1, 103.6, 99.6 (acetylenic $\underline{\mathrm{C}}$ ), 99.5 (acetylenic $\underline{\mathrm{C}}), 19.2\left(\mathrm{SCH}_{3}\right),-0.4\left(\mathrm{Si}\left(\underline{\mathrm{CH}}_{3}\right)_{3}\right.$; FTIR (neat) $3060,2958,2127,1573,1525,1494,1449 \mathrm{~cm}^{-1}$; APCI-MS $m / z$ calcd for $\mathrm{C}_{30} \mathrm{H}_{32} \mathrm{~S}_{4} \mathrm{Si}_{2}$ 576.1, found $577.0\left(100,[\mathrm{M}+\mathrm{H}]^{+}\right)$. 


\section{Synthesis of (3-(10-(4,5-bis(decylthio)-1,3-dithiol-2-ylidene)anthracen-9(10H)-ylid- ene)penta-1,4-diyne-1,5-diyl)bis(trimethylsilane) (6b).}

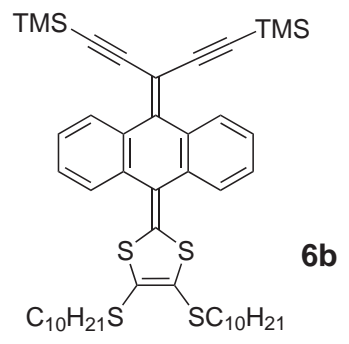

Compound $\mathbf{6} \mathbf{b}$ was prepared using the similar procedures as described in the synthesis of 6a: 5b (400 mg, $0.503 \mathrm{mmol})$, trimethylsilylacetylene (2.0 mL, $14 \mathrm{mmol}), \mathrm{PdCl}_{2}\left(\mathrm{PPh}_{3}\right)_{2}($ $18 \mathrm{mg}, 0.026 \mathrm{mmol})$, CuI ( $14 \mathrm{mg}, 0.074 \mathrm{mmol})$ in $\mathrm{Et}_{3} \mathrm{~N}(30 \mathrm{~mL})$. Pure $6 \mathbf{b}(400 \mathrm{mg}, 0.482$ mmol, 96\%) was afforded after silica column chromatography as a red-brown oil. ${ }^{1} \mathrm{H}$ NMR $\left(500 \mathrm{MHz}, \mathrm{CDCl}_{3}\right): \delta 8.27(\mathrm{~d}, J=8.0 \mathrm{~Hz}, 2 \mathrm{H}), 7.62(\mathrm{~d}, J=7.5 \mathrm{~Hz}, 2 \mathrm{H}), 7.34(\mathrm{t}, J=7.5$ $\mathrm{Hz}, 2 \mathrm{H}), 7.21$ (t, $J=7.5 \mathrm{~Hz}, 2 \mathrm{H}), 2.88-2.75(\mathrm{~m}, 4 \mathrm{H}), 1.66-1.60(\mathrm{~m}, 4 \mathrm{H}), 1.43-1.35(\mathrm{~m}, 4 \mathrm{H})$, $1.32-1.21(\mathrm{~m}, 24 \mathrm{H}), 0.87(\mathrm{t}, J=7.0 \mathrm{~Hz}, 6 \mathrm{H}), 0.22(\mathrm{~s}, 18 \mathrm{H}) ;{ }^{13} \mathrm{C} \mathrm{NMR}\left(125 \mathrm{MHz}, \mathrm{CDCl}_{3}\right): \delta$

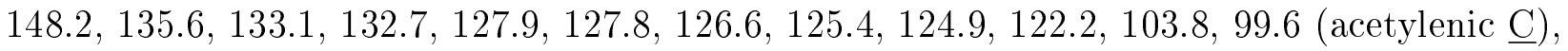
99.2 (acetylenic $\underline{\mathrm{C}}), 36.4,31.9,29.7,29.55,29.54,29.3,29.2,28.6,22.7,14.1,-0.4\left(\mathrm{Si}\left(\underline{\mathrm{CH}}_{3}\right)_{3}\right.$; FTIR (neat) $3060,2924,2853,1491,1458 \mathrm{~cm}^{-1}$; MALDI-TOF MS $\mathrm{m} / z$ calcd for $\mathrm{C}_{48} \mathrm{H}_{68} \mathrm{~S}_{4} \mathrm{Si}_{3}$ 828.37, found $829.40[\mathrm{M}+\mathrm{H}]^{+}$.

\section{Synthesis of exTTF $1 b$.}

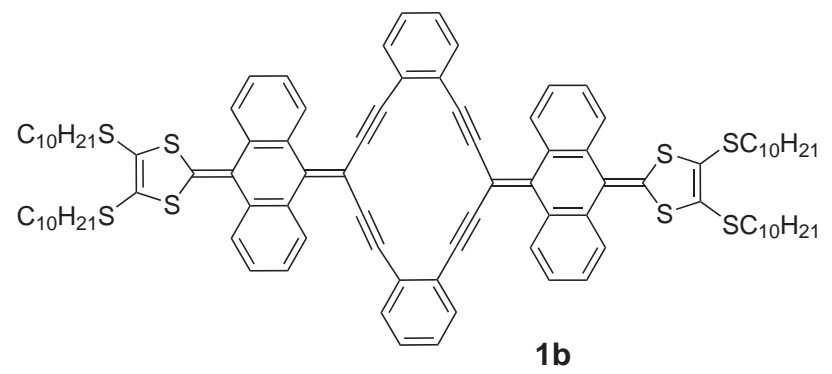

Compound 6b (133 mg, $0.160 \mathrm{mmol})$ was dissolved in THF $/ \mathrm{MeOH}(20 \mathrm{~mL}, 1: 1) \cdot \mathrm{K}_{2} \mathrm{CO}_{3}$ was added and the mixture was stirred for $30 \mathrm{~min}$ at $\mathrm{rt}$. The mixture was diluted with $\mathrm{Et}_{2} \mathrm{O}$, washed with $\mathrm{H}_{2} \mathrm{O}$, dried over $\mathrm{MgSO}_{4}$, and concentrated in vacuo to ca. $10 \mathrm{~mL}$. To a $100 \mathrm{~mL}$ round-bottom flask were charged with 1,2-diiodo benzene (50 mg, $0.15 \mathrm{mmol}$ ), CuI (12 $\mathrm{mg}, 0.063 \mathrm{mmol})$ and $\mathrm{Et}_{3} \mathrm{~N}(40 \mathrm{~mL})$, rigorous degassing was performed through three freeze-pump-thaw cycles. Then $\mathrm{Pd}\left(\mathrm{PPh}_{3}\right)_{4}(20 \mathrm{mg}, 0.017 \mathrm{mmol})$ was added under nitrogen protection, and the mixture was heated to $65{ }^{\circ} \mathrm{C}$. The residual solution containing desilylated $\mathbf{6 b}$ was purged with nitrogen bubbling for $5 \mathrm{~min}$, and then added into the mixture drop-wise 
over a period of $3 \mathrm{~h}$. The mixture was stirred overnight, then it was diluted with $\mathrm{CH}_{2} \mathrm{Cl}_{2}$, washed with aq. $\mathrm{NH}_{4} \mathrm{Cl}$ and $\mathrm{H}_{2} \mathrm{O}$, dried over $\mathrm{MgSO}_{4}$, and chromatographied with $10 \% \mathrm{CH}_{2} \mathrm{Cl}_{2}$ in hexanes as the eluent, affording the crude product of $\mathbf{1} \mathbf{b}$. Crude $\mathbf{1} \mathbf{b}$ was further purified by recrystallization from $\mathrm{MeOH} / \mathrm{CHCl}_{3}(1: 1, \mathrm{v} / \mathrm{v})$ to give pure $\mathbf{1 b}(32 \mathrm{mg}, 0.021 \mathrm{mmol}, 26 \%)$ as a black-red solid. m.p. $140-142{ }^{\circ} \mathrm{C} ;{ }^{1} \mathrm{H}$ NMR $\left(500 \mathrm{MHz}, \mathrm{CDCl}_{3}\right): \delta 8.45$ (dd, $J=11.5$, $7.0 \mathrm{~Hz}, 4 \mathrm{H}), 7.71$ (d, $J=5.0 \mathrm{~Hz}, 4 \mathrm{H}), 7.45-7.36(\mathrm{~m}, 12 \mathrm{H}), 7.31-7.26(\mathrm{~m}, 4 \mathrm{H}), 2.88-2.73$ $(\mathrm{m}, 8 \mathrm{H}), 1.65-1.56(\mathrm{~m}, 8 \mathrm{H}), 1.42-1.33(\mathrm{~m}, 8 \mathrm{H}), 1.28-1.16(\mathrm{~m}, 48 \mathrm{H}), 0.86-0.80(\mathrm{~m}, 12 \mathrm{H}) ;{ }^{13} \mathrm{C}$ NMR (125 MHz, $\left.\mathrm{CDCl}_{3}\right): \delta$ 135.9, 133.2, 131.9, 128.1, 127.9, 126.7, 125.51, 125.46, 125.1, 99.6 (four coincidental sp $\mathrm{sp}^{2}$ carbon peaks not observed), 91.7 (acetylenic $\underline{\mathrm{C}}$ ), 86.6 (acetylenic C) , 36.5, 31.9, 29.6, 29.52, 29.50, 29.3, 29.1, 28.5, 22.7, 14.1 ( $\left.\underline{\mathrm{SCH}}_{3}\right)$; FTIR (neat) 3060, 2958, 2924, 2853, 1572, 1531, $1485 \mathrm{~cm}^{-1}$; MALDI-TOF MS m/z calcd for $\mathrm{C}_{96} \mathrm{H}_{108} \mathrm{~S}_{8}, 1516.62$ found $1518.82[\mathrm{M}+\mathrm{H}]^{+}$. X-ray structure determination. ${ }^{3}$

\section{Synthesis of compounds 7 and 8 .}

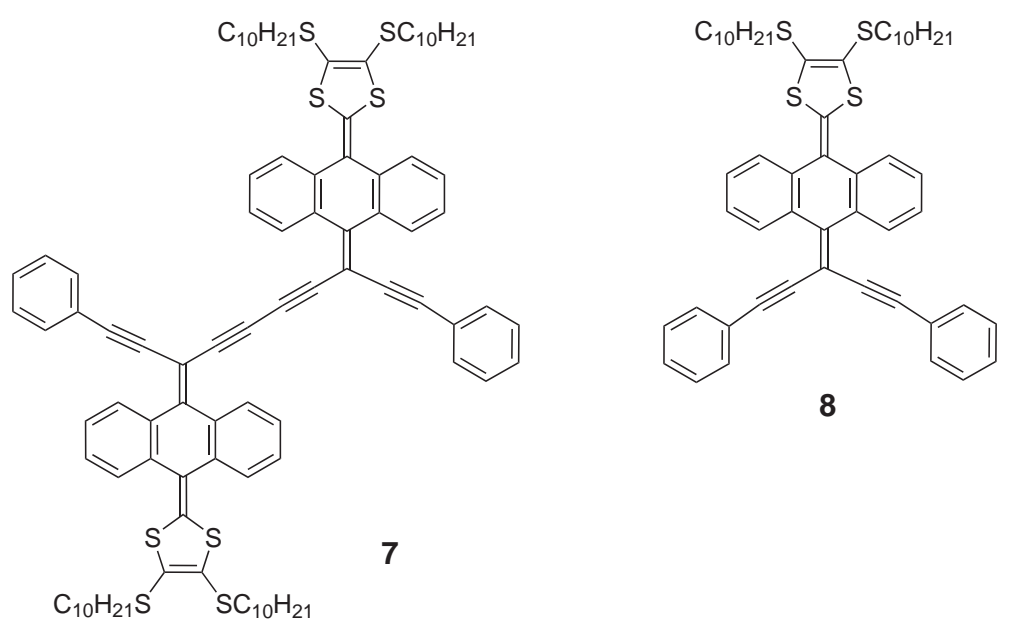

Method 1: Compound 6b (38 mg, $0.046 \mathrm{mmol})$ was dissolved in $\mathrm{THF} / \mathrm{MeOH}(15 \mathrm{~mL}$, 1:1). Excess $\mathrm{K}_{2} \mathrm{CO}_{3}$ was added to the solution, and the mixture was kept stirring for $30 \mathrm{~min}$. The mixture was diluted with $\mathrm{Et}_{2} \mathrm{O}$, washed with $\mathrm{H}_{2} \mathrm{O}$, dried over $\mathrm{MgSO}_{4}$, and concentrated in vacuo to $c a$. $5 \mathrm{~mL}$. To a $50 \mathrm{~mL}$ round-bottom flask were charged with iodobenzene $(9.5$ $\mathrm{mg}, 0.047 \mathrm{mmol})$, CuI (3 mg, $0.016 \mathrm{mmol})$, and $\mathrm{Et}_{3} \mathrm{~N}(15 \mathrm{~mL})$. The mixture was rigorously degassed by three freeze-pump-thaw cycles, then $\mathrm{Pd}\left(\mathrm{PPh}_{3}\right)_{4}(5 \mathrm{mg}, 0.004 \mathrm{mmol})$, and degassed residual solution of desilylated $\mathbf{6 b}$ was added. The mixture was stirred overnight, and then was diluted with $\mathrm{CH}_{2} \mathrm{Cl}_{2}$, washed with aq. $\mathrm{NH}_{4} \mathrm{Cl}$ and $\mathrm{H}_{2} \mathrm{O}$, dried over $\mathrm{MgSO} 4$, and chromatographied using $5 \% \mathrm{CH}_{2} \mathrm{Cl}_{2}$ in hexanes, affording compound 8 (10 mg, $0.012 \mathrm{mmol}$, $51 \%)$ as an orange oil, and compound 7 (14 $\mathrm{mg}, 0.0093 \mathrm{mmol}, 40 \%)$ as a red oil .

\footnotetext{
${ }^{3}$ For detailed crystallographic data, see attached CIF document.
} 
Method 2: Compound 6b (31 mg, $0.037 \mathrm{mmol})$ was desilylated and then cross-coupled with iodobenzene $(0.1 \mathrm{~mL}, 0.9 \mathrm{mmol})$ in the presence of $\mathrm{PdCl}_{2}\left(\mathrm{PPh}_{3}\right)_{2}(5 \mathrm{mg}, 0.007 \mathrm{mmol})$, $\mathrm{CuI}(3 \mathrm{mg}, 0.02 \mathrm{mmol})$, and $\mathrm{Et}_{3} \mathrm{~N}(20 \mathrm{~mL})$, using a similar procedure as described in Method 1. Compound 8 (18 mg, $0.021 \mathrm{mmol}, 58 \%)$ was obtained as the major product, whereas the yield of compound 7 was trace under these conditions.

Compound 7. ${ }^{1} \mathrm{H}$ NMR $\left(500 \mathrm{MHz}, \mathrm{CDCl}_{3}\right): \delta 8.38(\mathrm{~d}, J=8.5 \mathrm{~Hz}, 2 \mathrm{H}), 7.68(\mathrm{t}, J=$ $8.0 \mathrm{~Hz}, 4 \mathrm{H}), 7.51-7.45(\mathrm{~m}, 4 \mathrm{H}), 7.42-7.36(\mathrm{~m}, 4 \mathrm{H}), 7.36-7.28(\mathrm{~m}, 10 \mathrm{H}), 2.89-2.70(\mathrm{~m}, 8 \mathrm{H})$, $1.67-1.58(\mathrm{~m}, 8 \mathrm{H}), 1.44-1.35(\mathrm{~m}, 8 \mathrm{H}), 1.30-1.20(\mathrm{~m}, 48 \mathrm{H}), 0.88-0.82(\mathrm{~m}, 12 \mathrm{H}) ;{ }^{13} \mathrm{C}$ NMR $\left(125 \mathrm{MHz} \mathrm{CDCl}_{3}\right): \delta 149.1,135.6,135.5,134.0,132.78,132.77,132.73,132.70,131.6,128.6$, 128.4, 128.1, 127.6, 126.69, 126.66, 126.1, 125.6, 125.1, 123.0, 121.9, 99.6 (one coincidental $\mathrm{sp}^{2}$ carbon peaks not observed), 98.6 (acetylenic $\underline{\mathrm{C}}$ ), 93.9 (acetylenic $\underline{\mathrm{C}}$ ), 88.2 (acetylenic $\underline{\mathrm{C}}$ ), 83.1 (acetylenic $\underline{\mathrm{C}}$ ), 36.444, 36.438, 31.9, 29.71, 29.70, 29.68, 29.57, 29.54, 29.52, 29.4, 29.314, 29.308, 29.18, 29.15, 28.54, 28.52, 22.7, 14.1; FTIR (neat) 3060, 2924, 2853, 1572, 1525, 1492 , $1457 \mathrm{~cm}^{-1}$; MALDI-TOF MS $\mathrm{m} / z$ calcd for $\mathrm{C}_{96} \mathrm{H}_{110} \mathrm{~S}_{8} 1518.64$, found $1520.33[\mathrm{M}+\mathrm{H}]^{+}$.

Compound 8. ${ }^{1} \mathrm{H}$ NMR (500 MHz, $\left.\mathrm{CDCl}_{3}\right): \delta 8.40(\mathrm{dd}, J=8.0 \mathrm{~Hz}, 1.5 \mathrm{~Hz}, 2 \mathrm{H}), 7.67(\mathrm{~d}$, $J=8.0 \mathrm{~Hz}, 2 \mathrm{H}), 7.52-7.47(\mathrm{~m}, 4 \mathrm{H}), 7.42-7.32(\mathrm{~m}, 10 \mathrm{H}), 2.86-2.75(\mathrm{~m}, 4 \mathrm{H}), 1.66-1.57$ (m, $4 \mathrm{H}), 1.42-1.32(\mathrm{~m}, 4 \mathrm{H}), 1.30-1.18(\mathrm{~m}, 24 \mathrm{H}), 0.86(\mathrm{t}, J=7.0 \mathrm{~Hz}, 6 \mathrm{H}) ;{ }^{13} \mathrm{C} \mathrm{NMR}(125 \mathrm{MHz}$, $\left.\mathrm{CDCl}_{3}\right): \delta 146.4,135.8,133.23,133.18,131.5,128.5,128.4,127.73,127.66,126.6,125.6,125.1$, 123.3, 122.2, 99.6, 93.1 (acetylenic $\underline{\mathrm{C}}$ ), 89.2 (acetylenic $\underline{\mathrm{C}}$ ), 36.4, 31.9, 29.7, 29.54, 29.52, 29.3, 29.2, 28.5, 22.7, 14.1; FTIR (neat) 3060, 2924, 2853, 1574, 1531, $1496 \mathrm{~cm}^{-1}$; MALDI-TOF MS $m / z$ calcd for $\mathrm{C}_{54} \mathrm{H}_{60} \mathrm{~S}_{4}$ 836.36, found $837.54[\mathrm{M}+\mathrm{H}]^{+}$.

\section{NMR Spectra of Compounds 4-8}




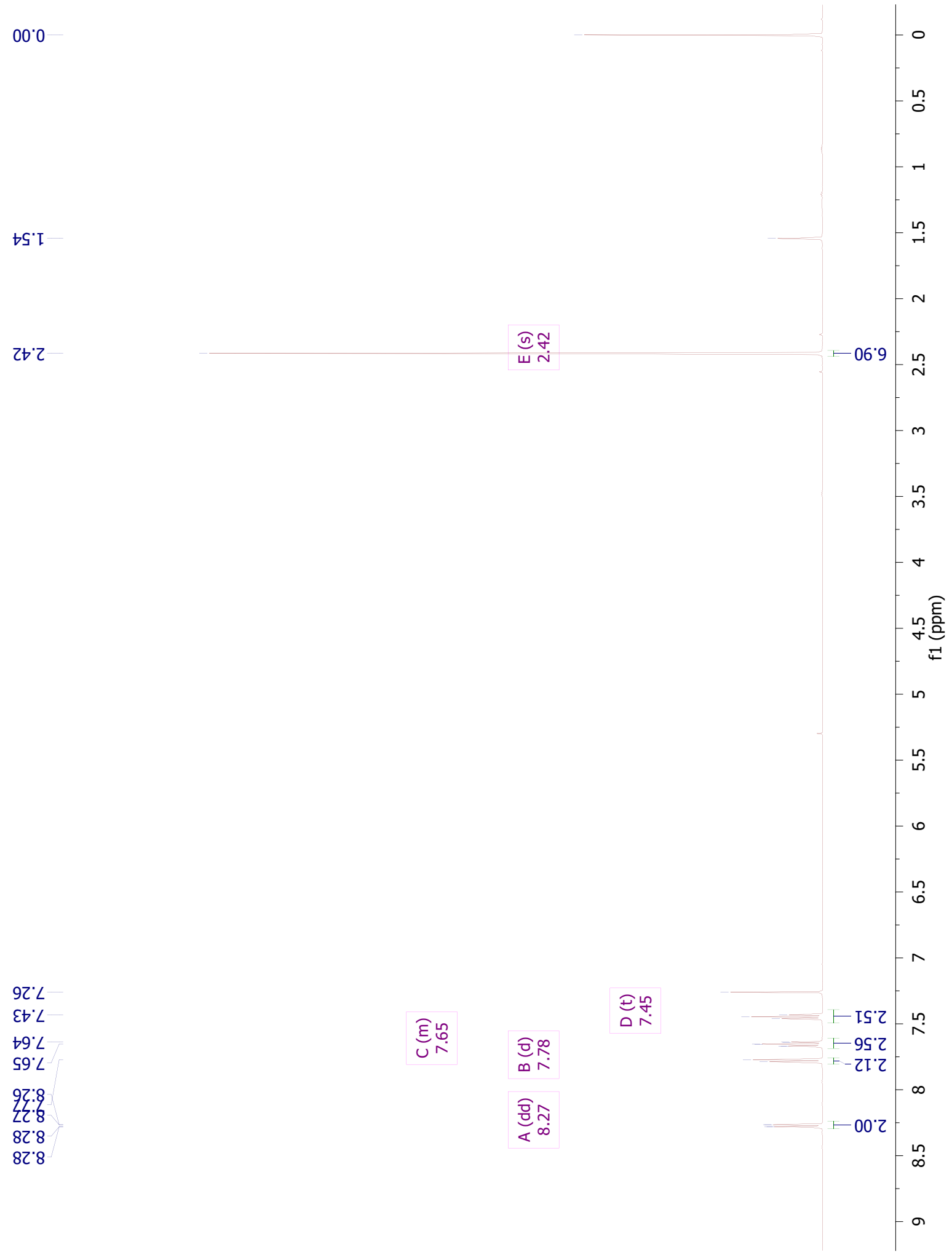

Fig. $\mathrm{S}-1:{ }^{1} \mathrm{H}$ NMR spectrum of $\mathbf{4 a}$. 


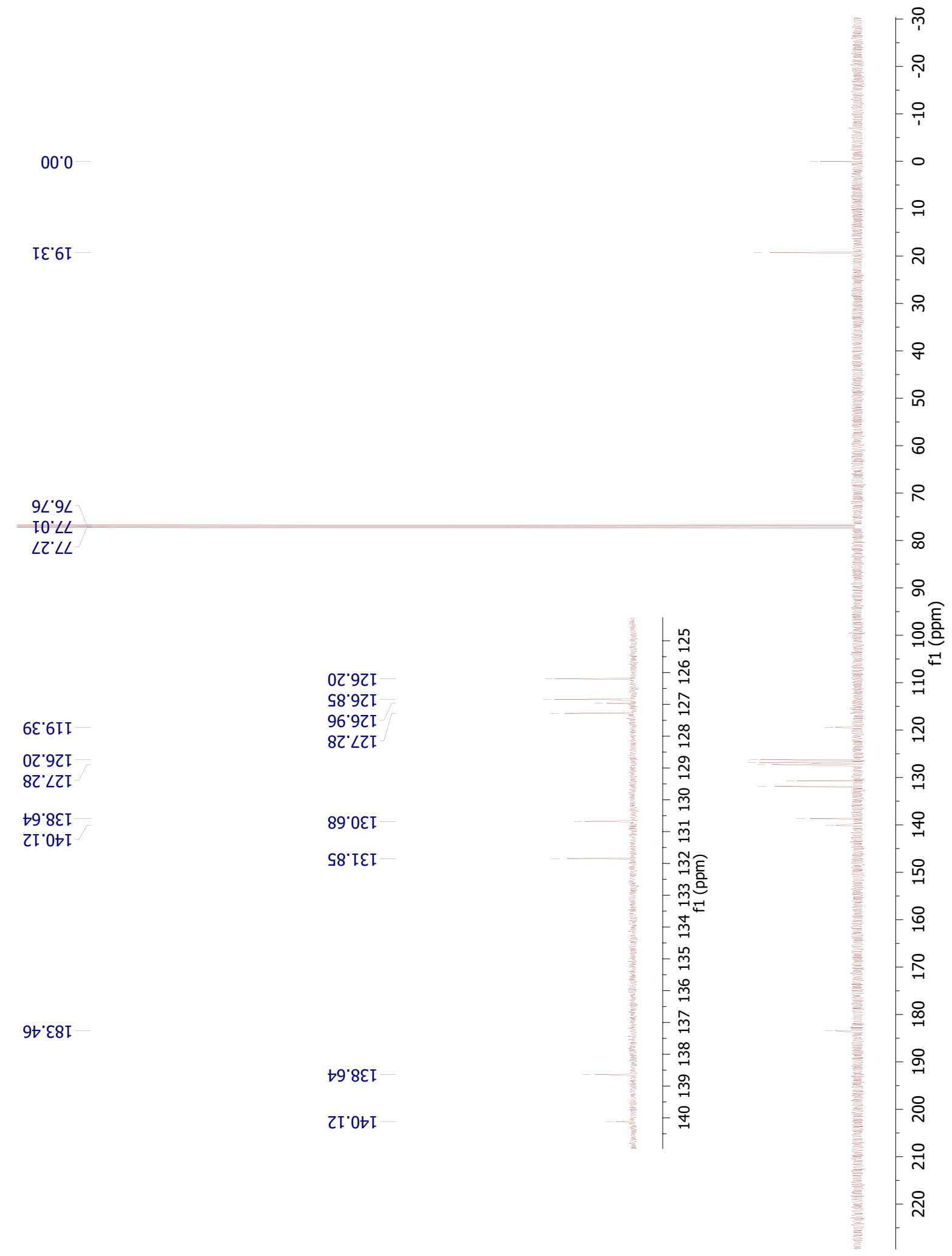

Fig. S $-2:{ }^{13} \mathrm{C}$ NMR spectrum of $\mathbf{4 a}$. 


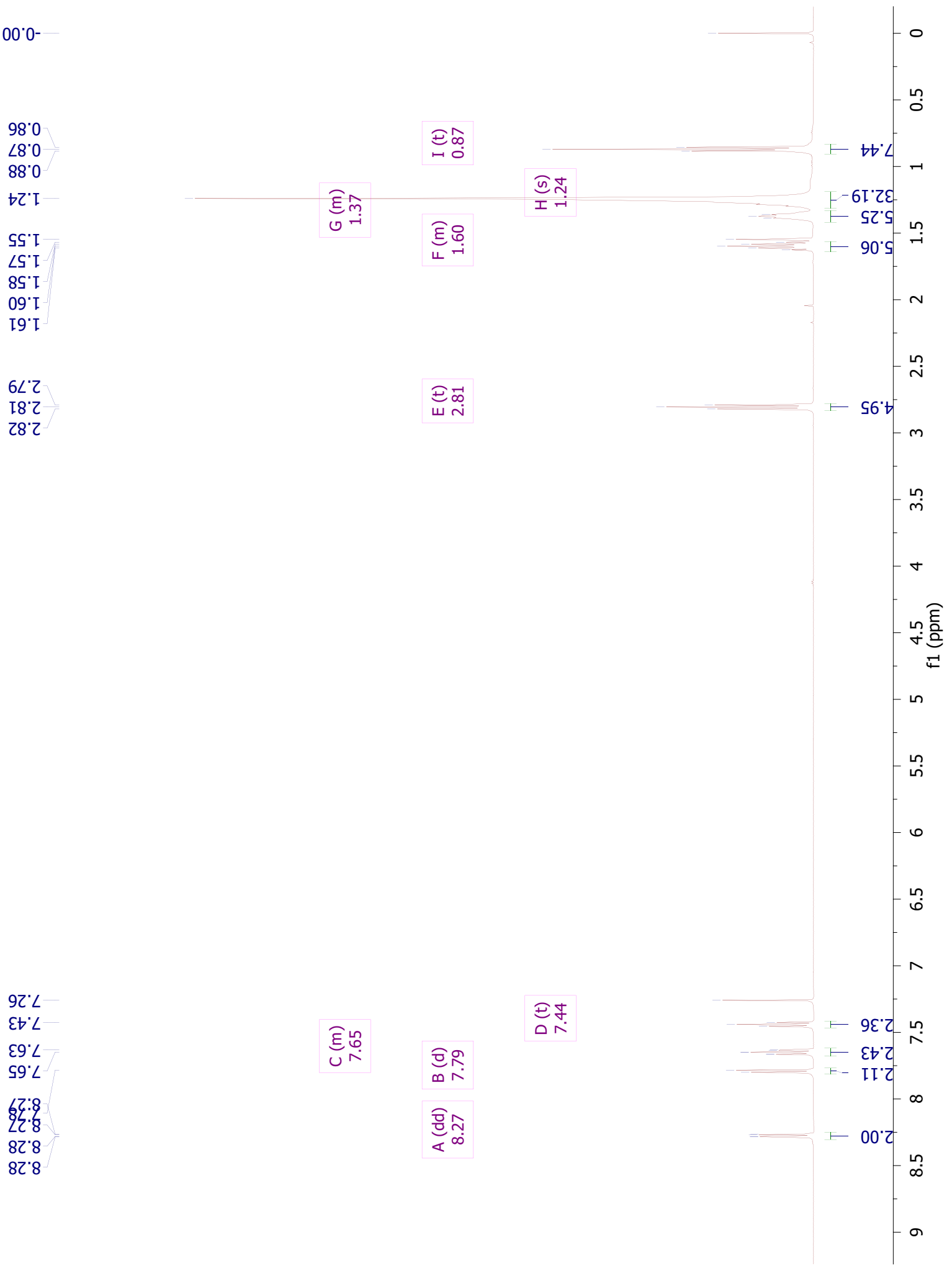

Fig. $\mathrm{S}-3:{ }^{1} \mathrm{H}$ NMR spectrum of $\mathbf{4} \mathbf{b}$. 


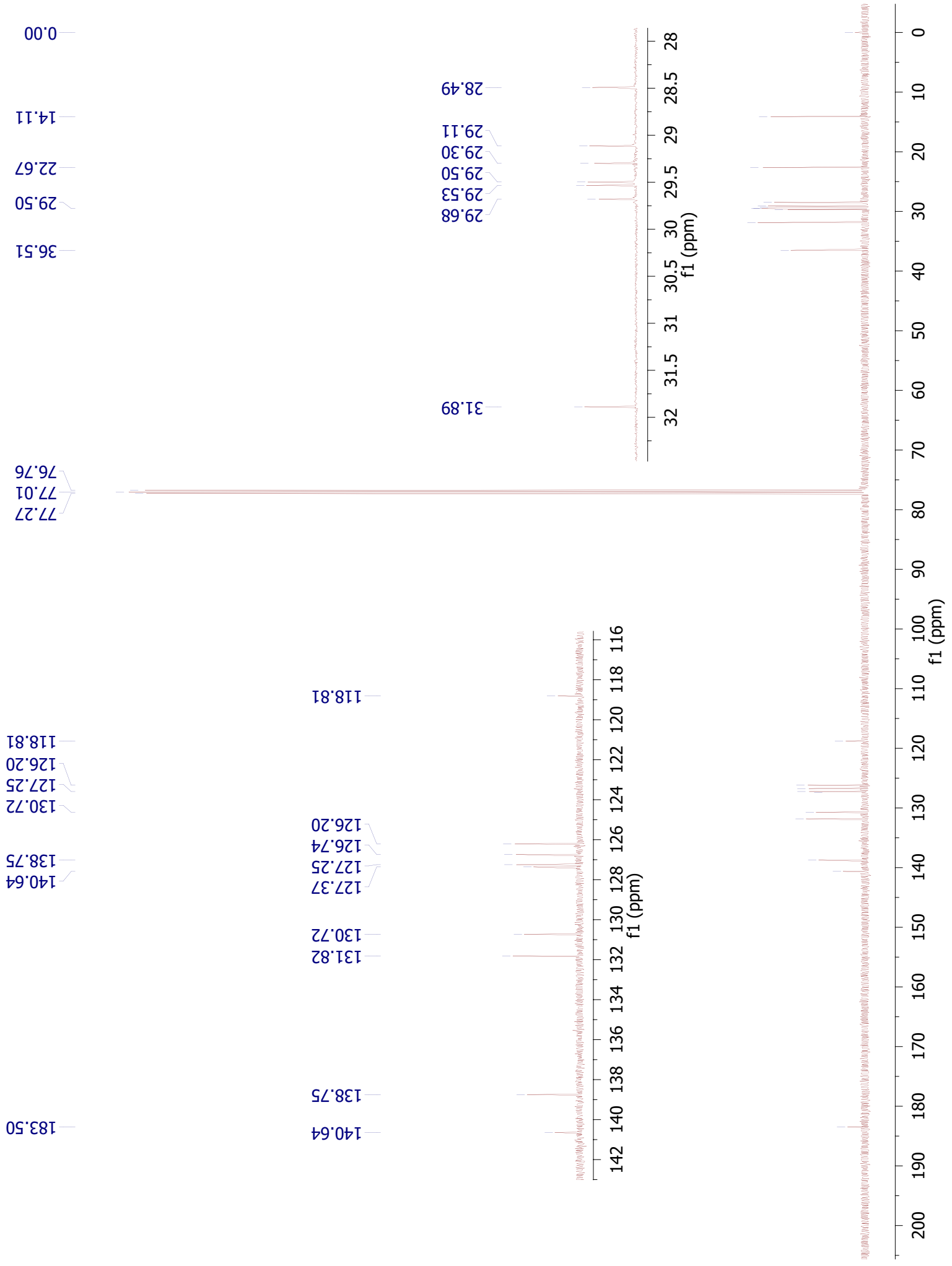

Fig. $\mathrm{S}-4:{ }^{13} \mathrm{C}$ NMR spectrum of $\mathbf{4 b}$. 


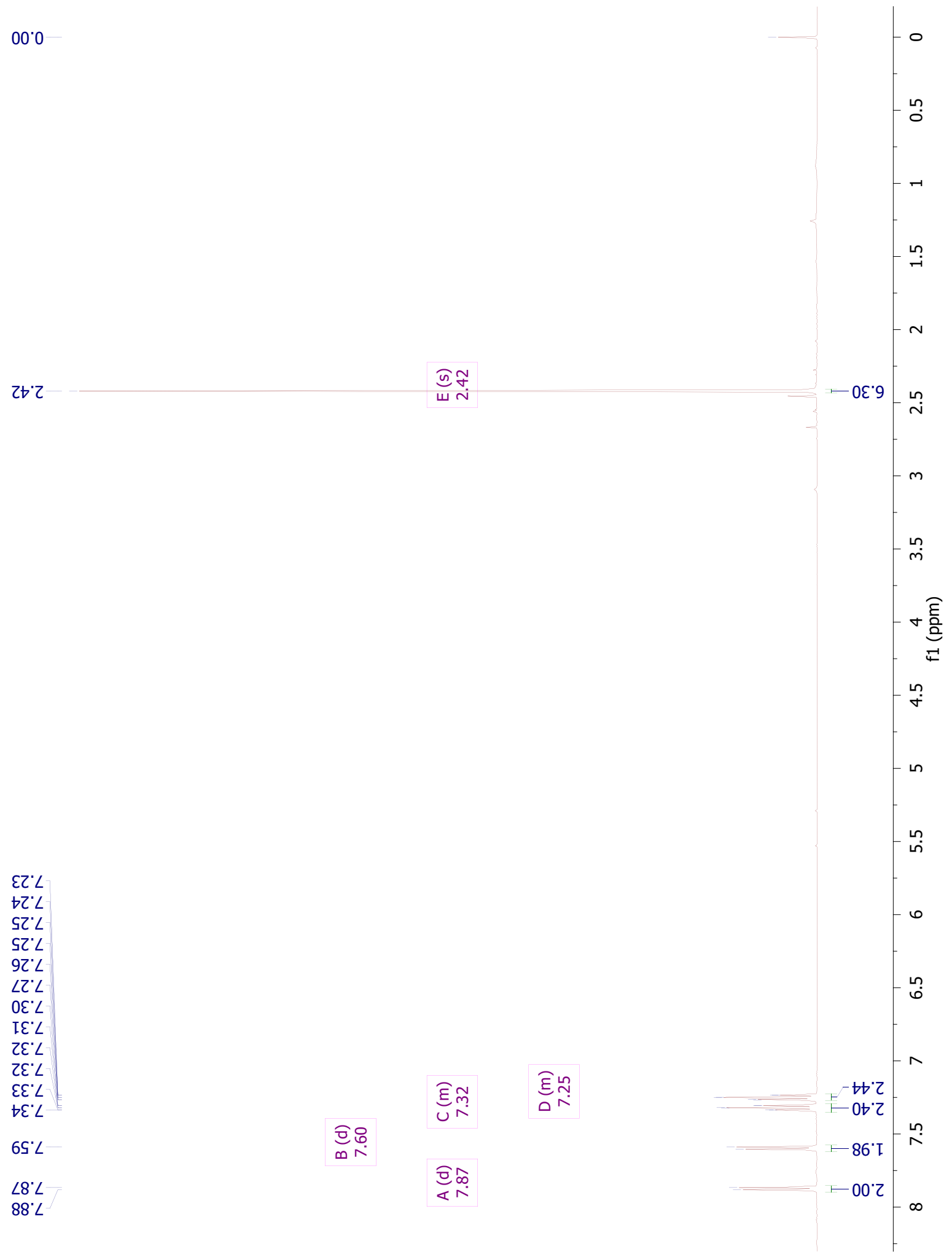

Fig. S $-5:{ }^{1} \mathrm{H}$ NMR spectrum of $\mathbf{5 a}$. 


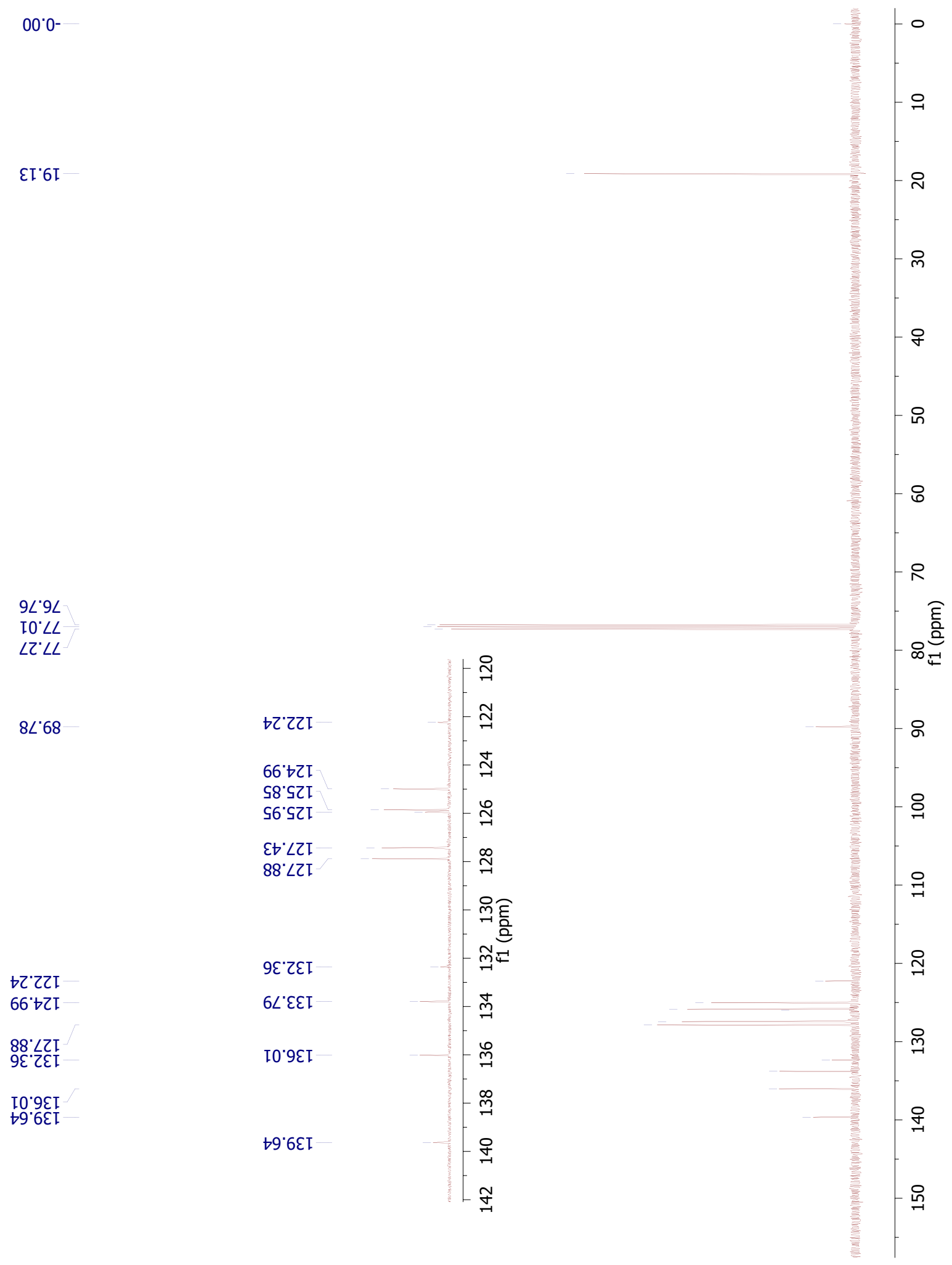

Fig. $\mathrm{S}-6:{ }^{13} \mathrm{C}$ NMR spectrum of $\mathbf{5 a}$. 


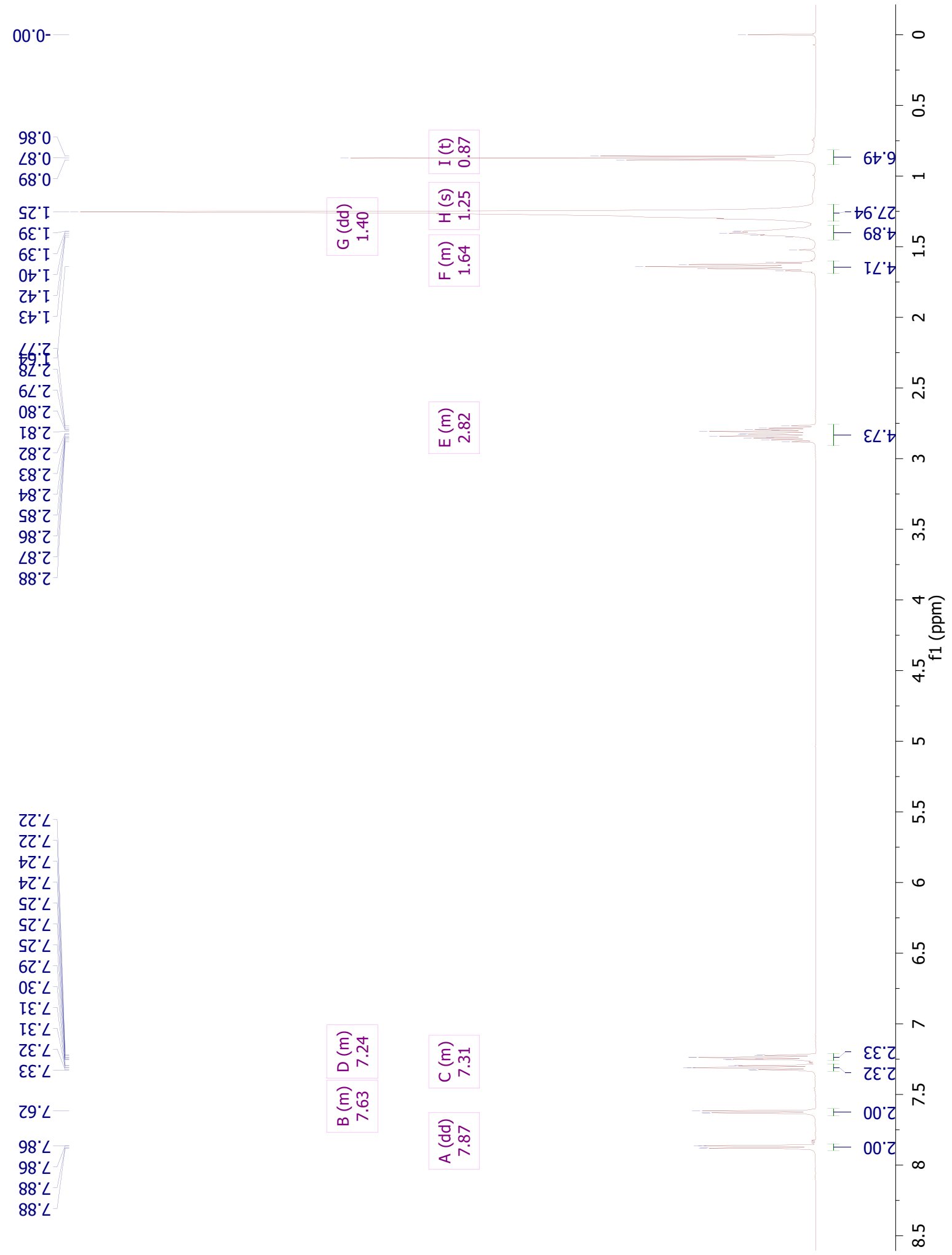

Fig. $\mathrm{S}-7:{ }^{1} \mathrm{H}$ NMR spectrum of $\mathbf{5} \mathbf{b}$. 

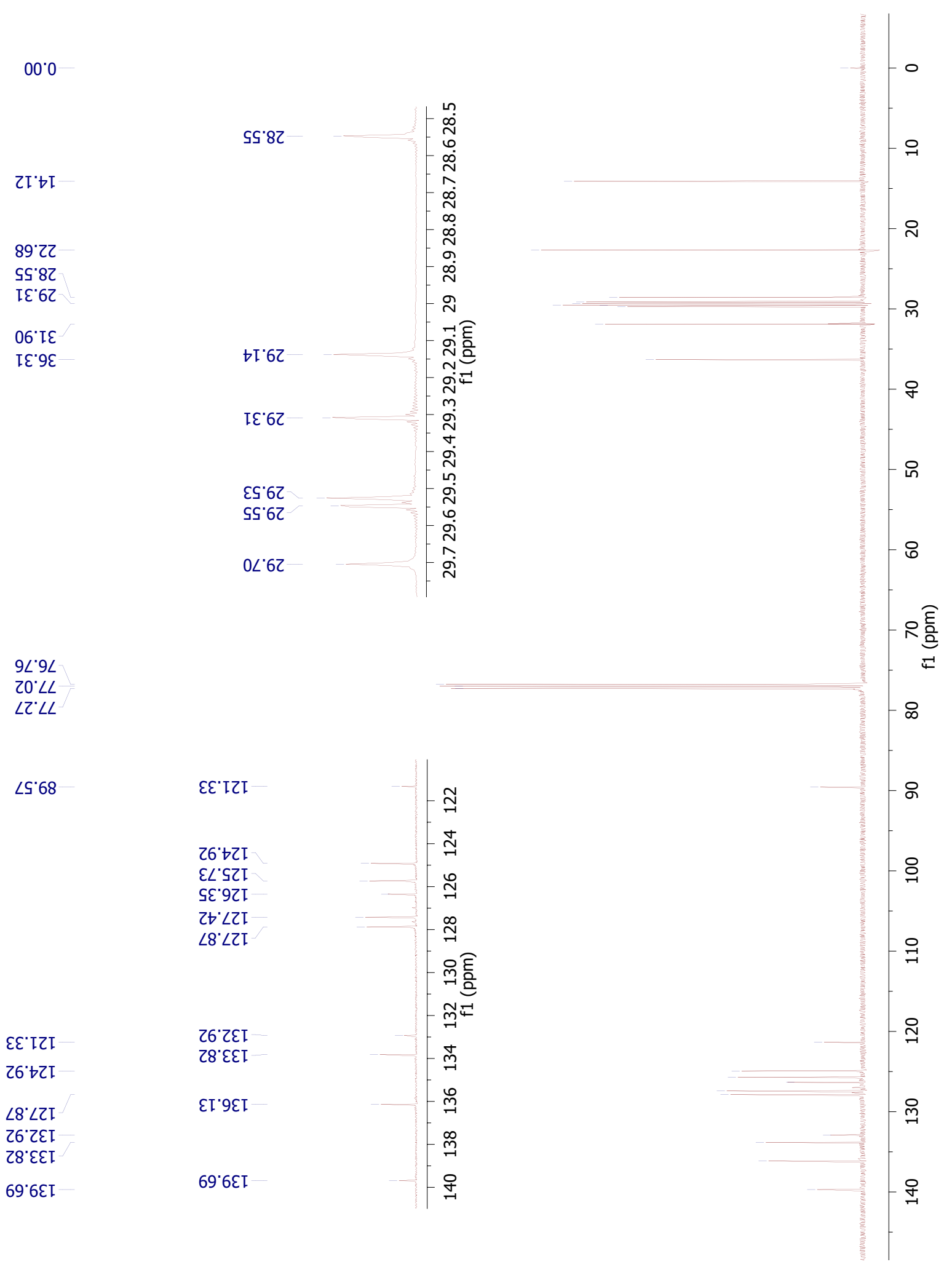

Fig. $\mathrm{S}-8:{ }^{13} \mathrm{C}$ NMR spectrum of $\mathbf{5} \mathbf{b}$. 


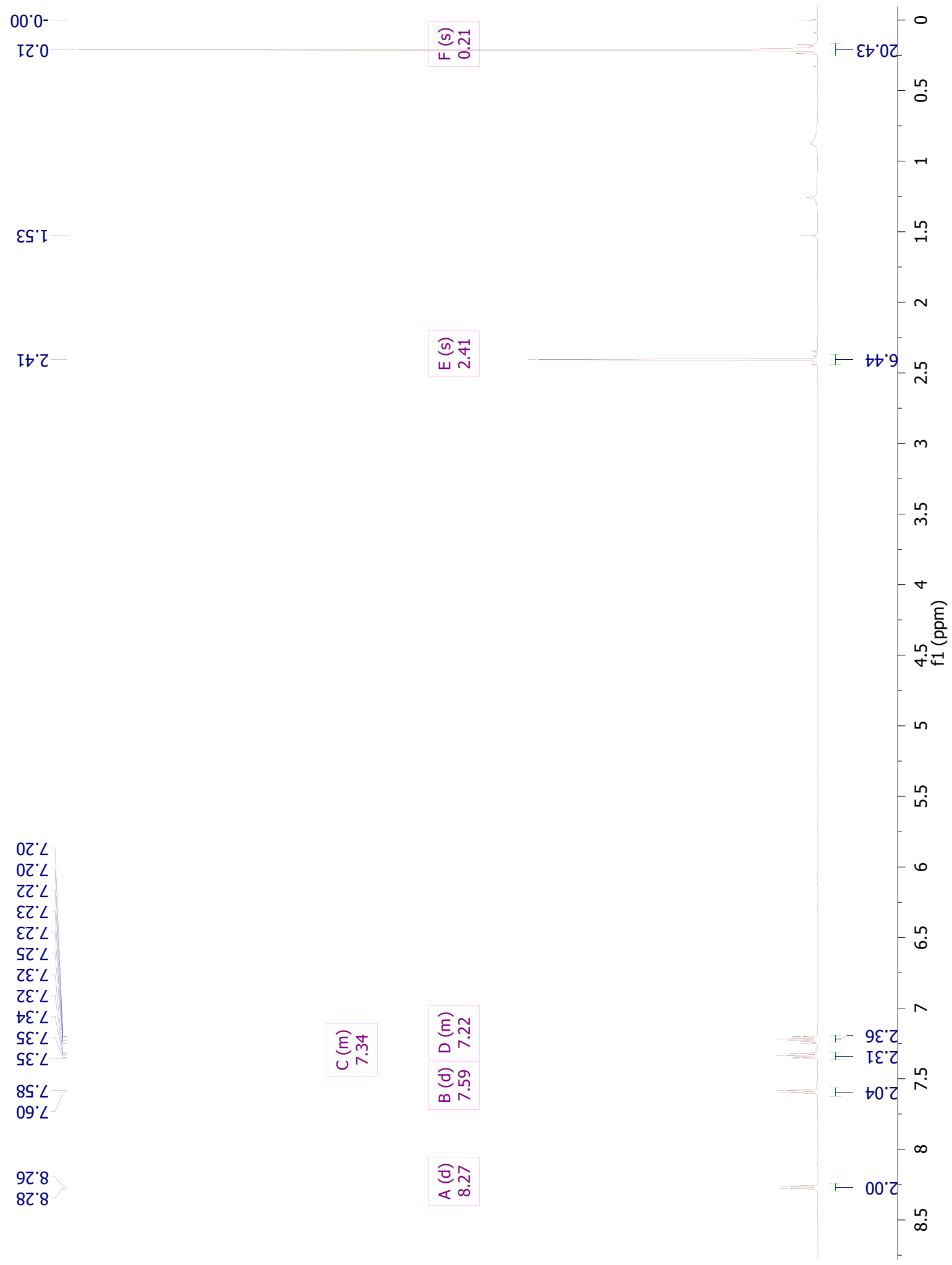

Fig. $\mathrm{S}-9:{ }^{1} \mathrm{H}$ NMR spectrum of $\mathbf{6 a}$. 


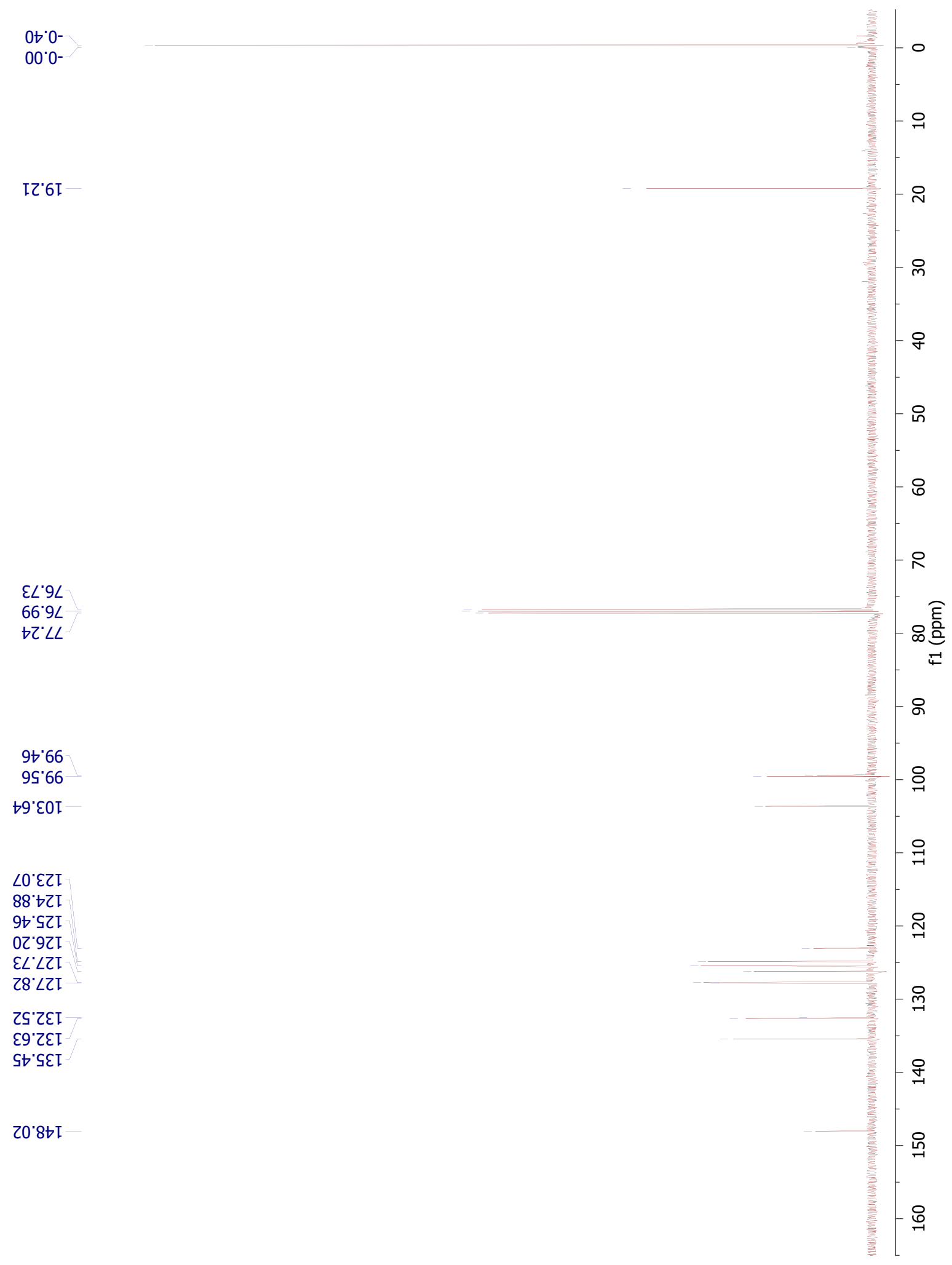

Fig. S $-10:{ }^{13} \mathrm{C}$ NMR spectrum of $\mathbf{6 a}$. 


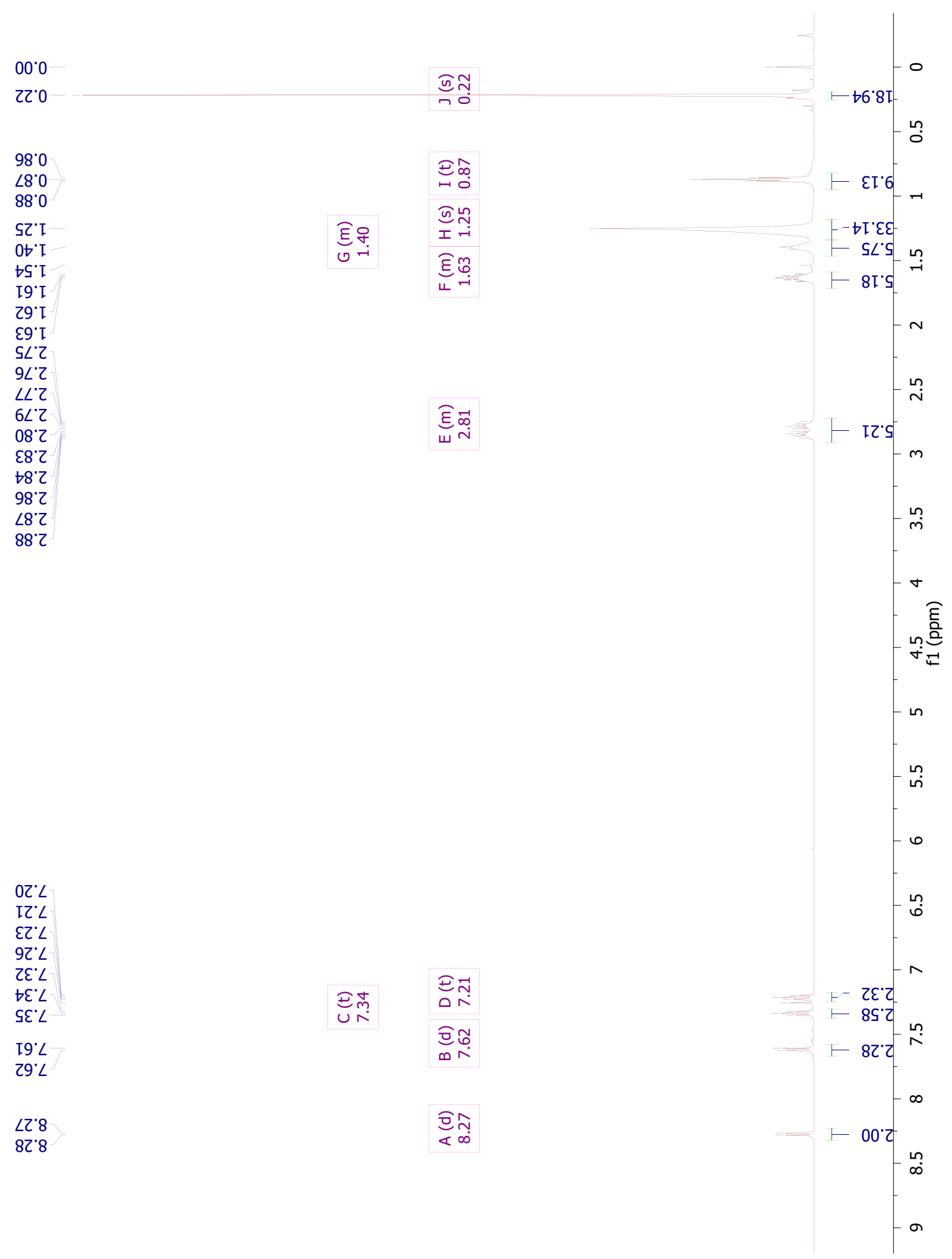

Fig. $\mathrm{S}-11:{ }^{1} \mathrm{H}$ NMR spectrum of $\mathbf{6} \mathbf{b}$. 


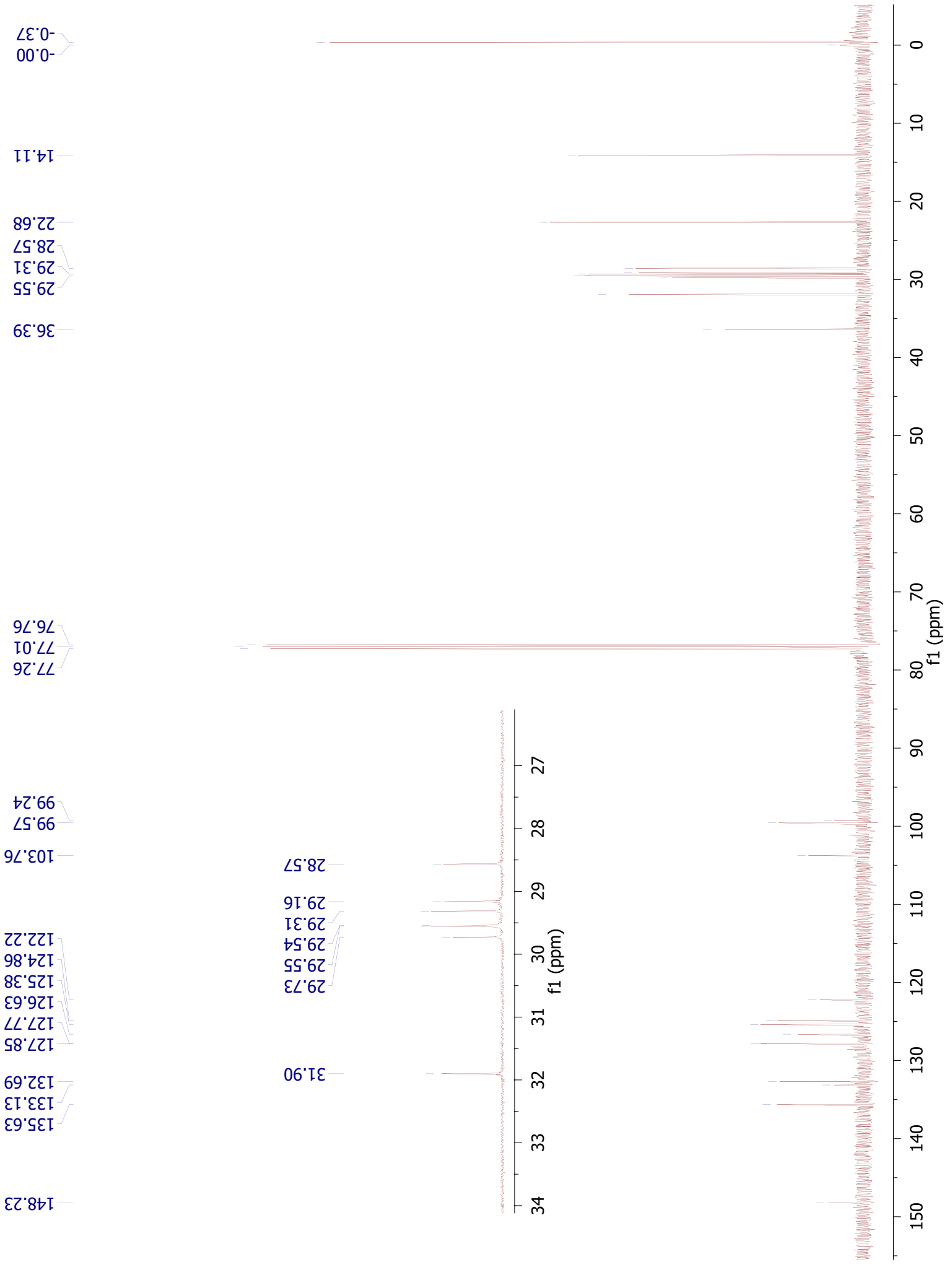

Fig. $\mathrm{S}-12:{ }^{13} \mathrm{C}$ NMR spectrum of $\mathbf{6} \mathbf{b}$. 


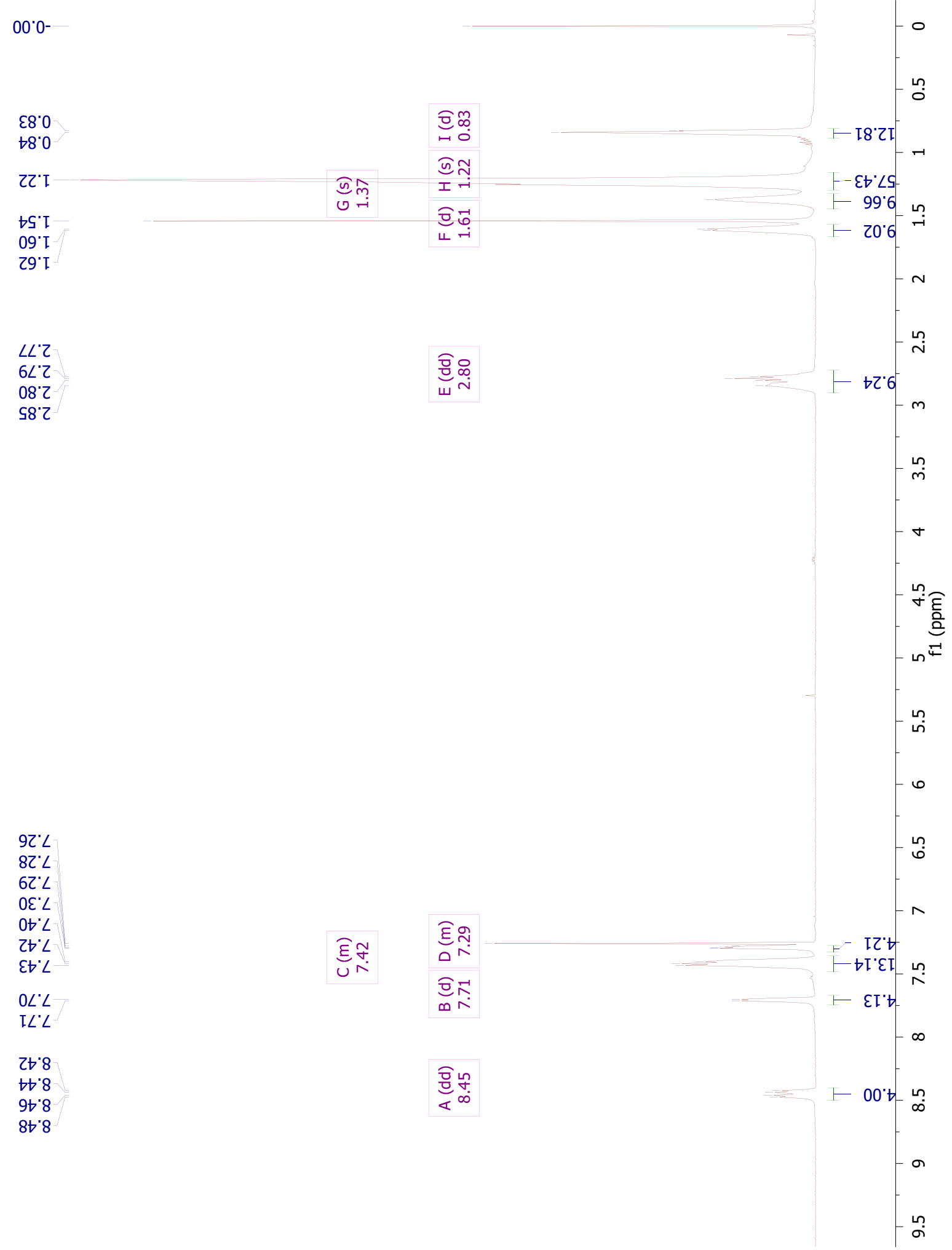

Fig. $\mathrm{S}-13:{ }^{1} \mathrm{H}$ NMR spectrum of $\mathbf{1} \mathbf{b}$. 


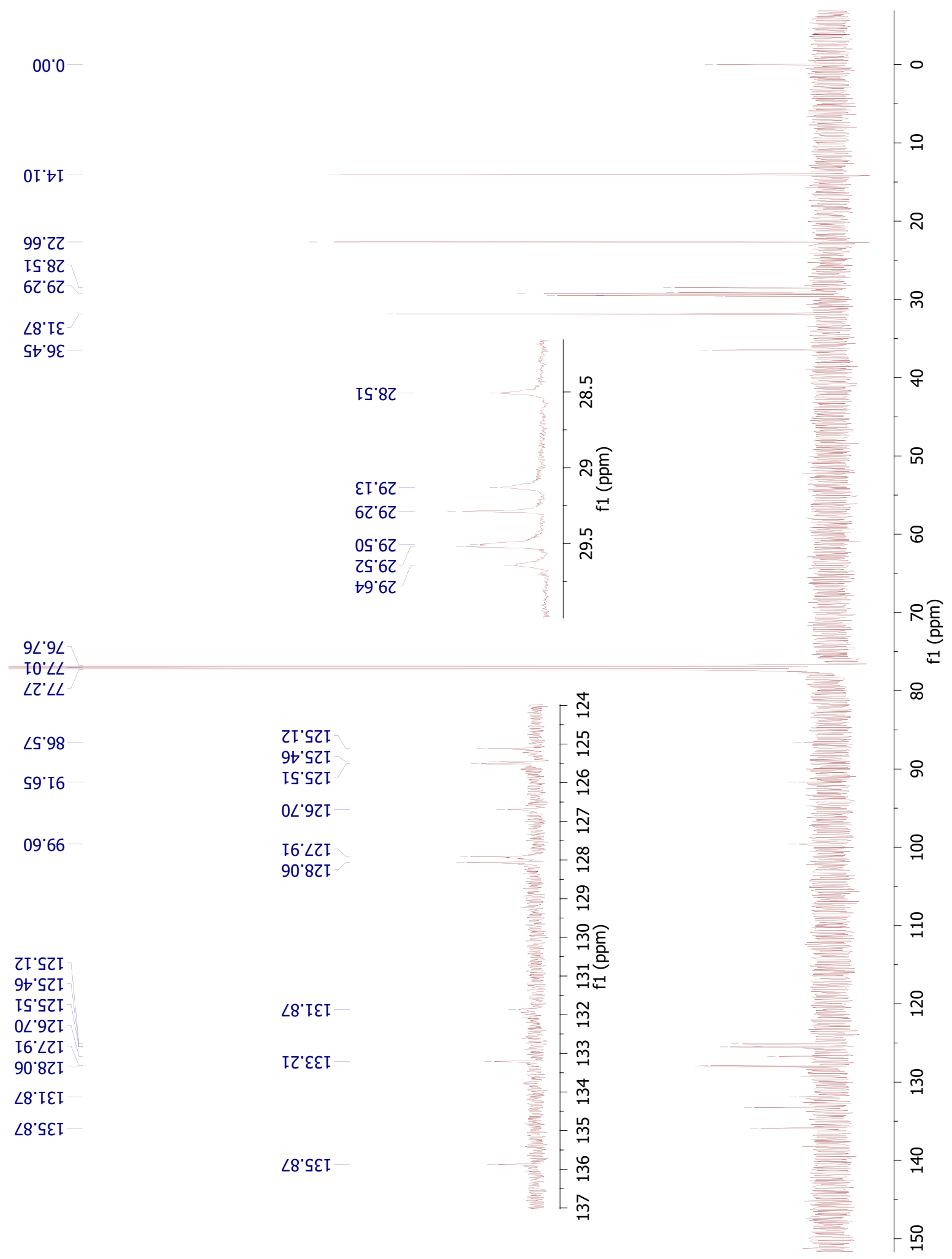

Fig. $\mathrm{S}-14:{ }^{13} \mathrm{C}$ NMR spectrum of $\mathbf{1 b}$. 


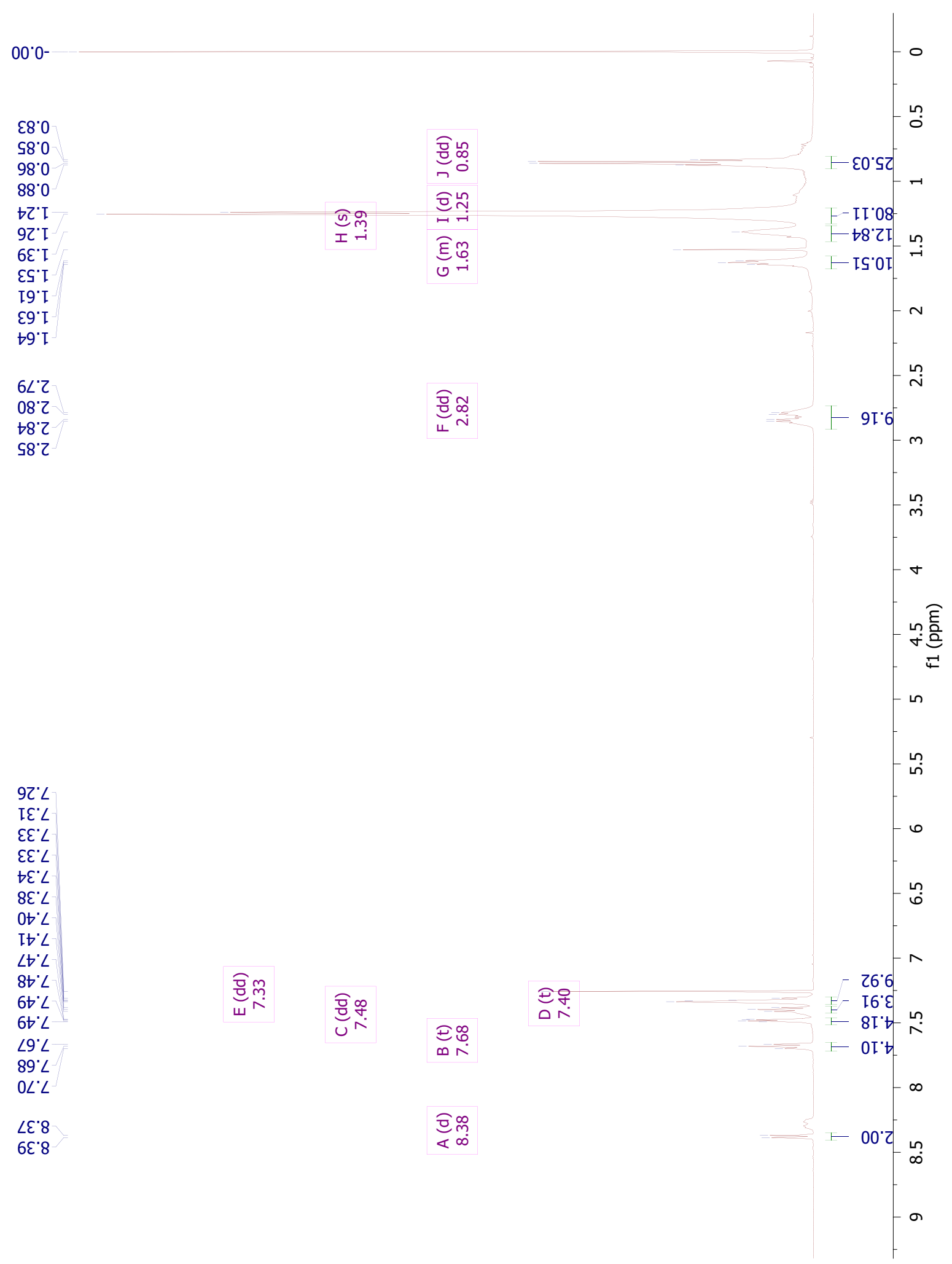

Fig. $\mathrm{S}-15:{ }^{1} \mathrm{H}$ NMR spectrum of 7 . 


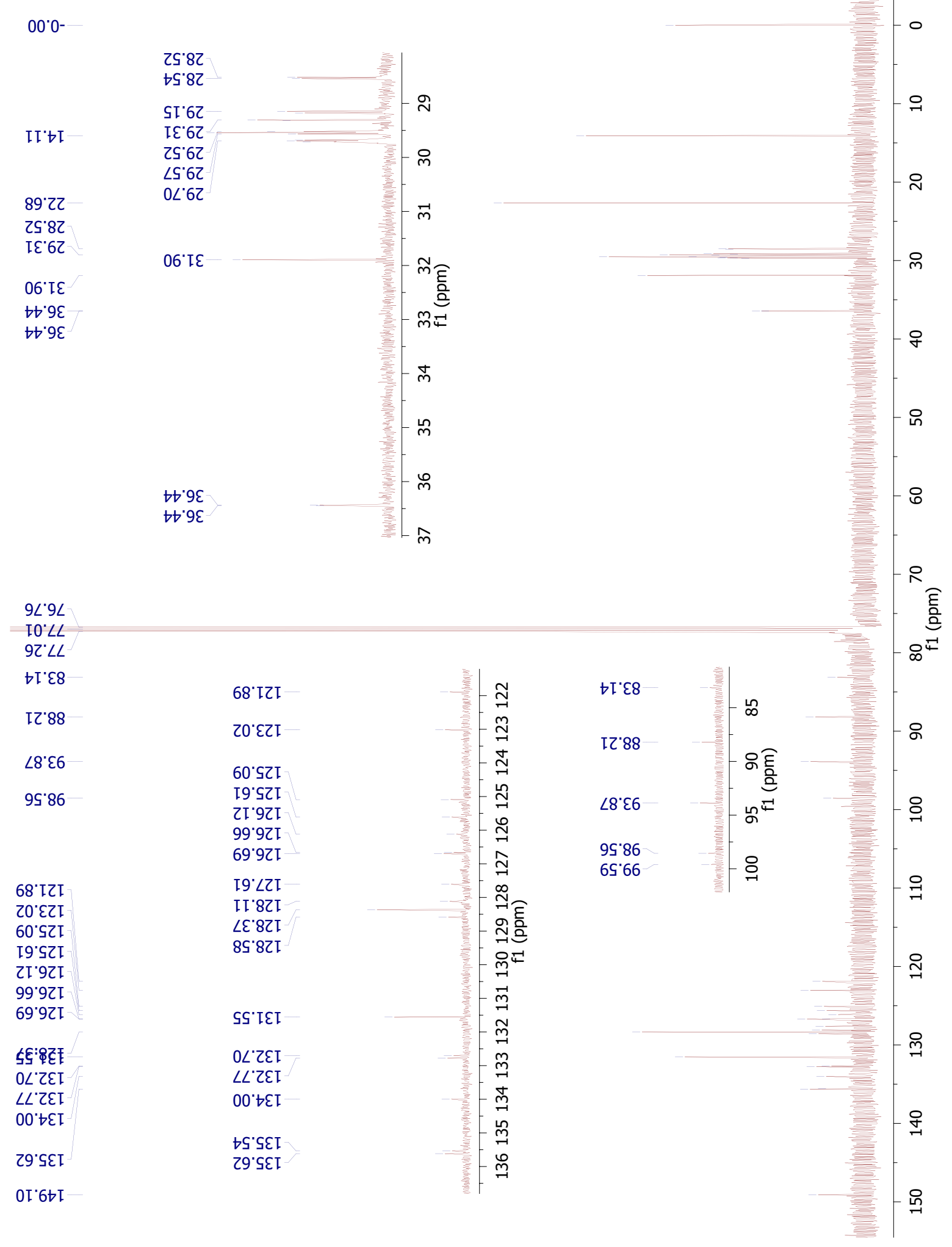

Fig. $\mathrm{S}-16:{ }^{13} \mathrm{C}$ NMR spectrum of 7 . 


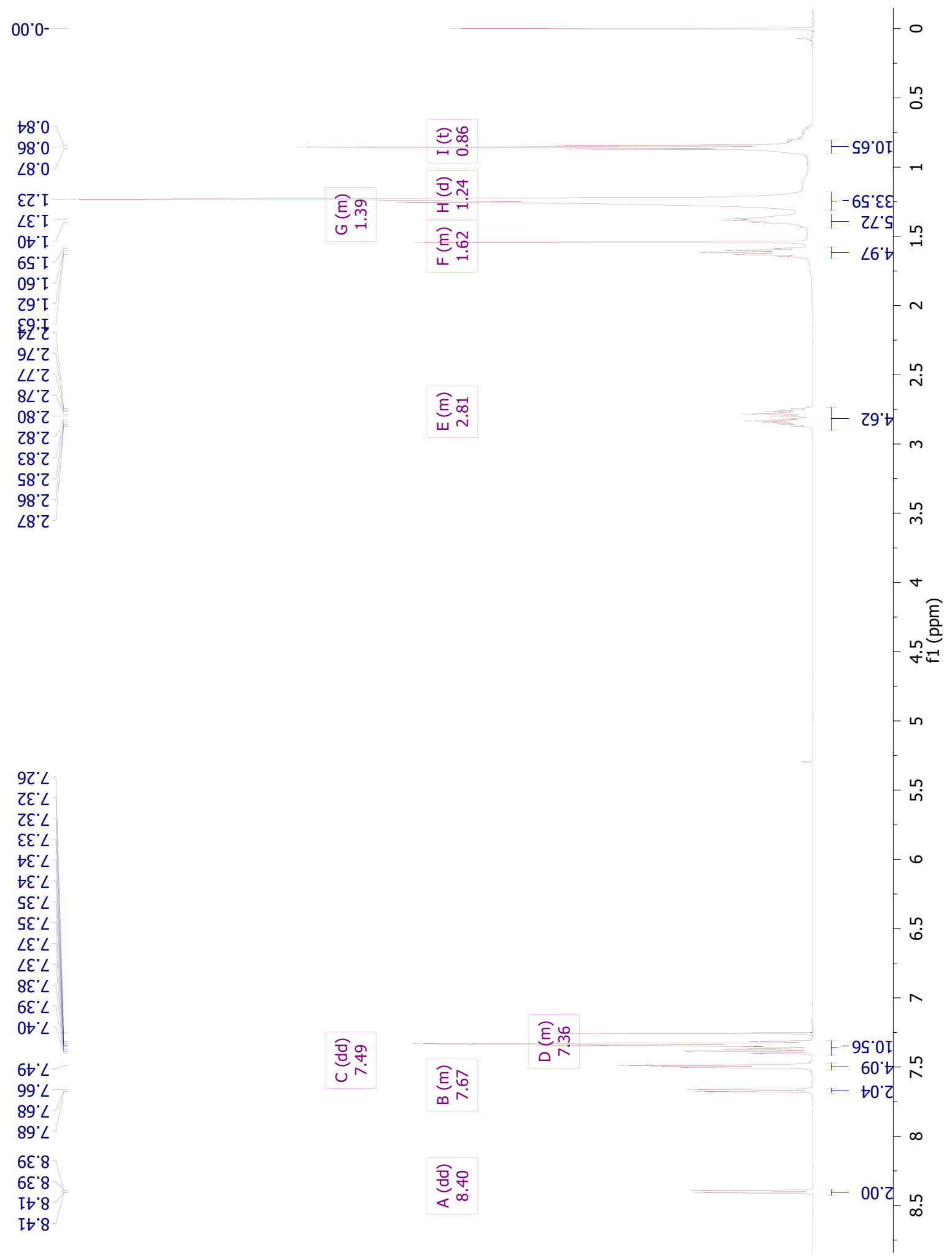

Fig. $\mathrm{S}-17:{ }^{1} \mathrm{H}$ NMR spectrum of 8 . 


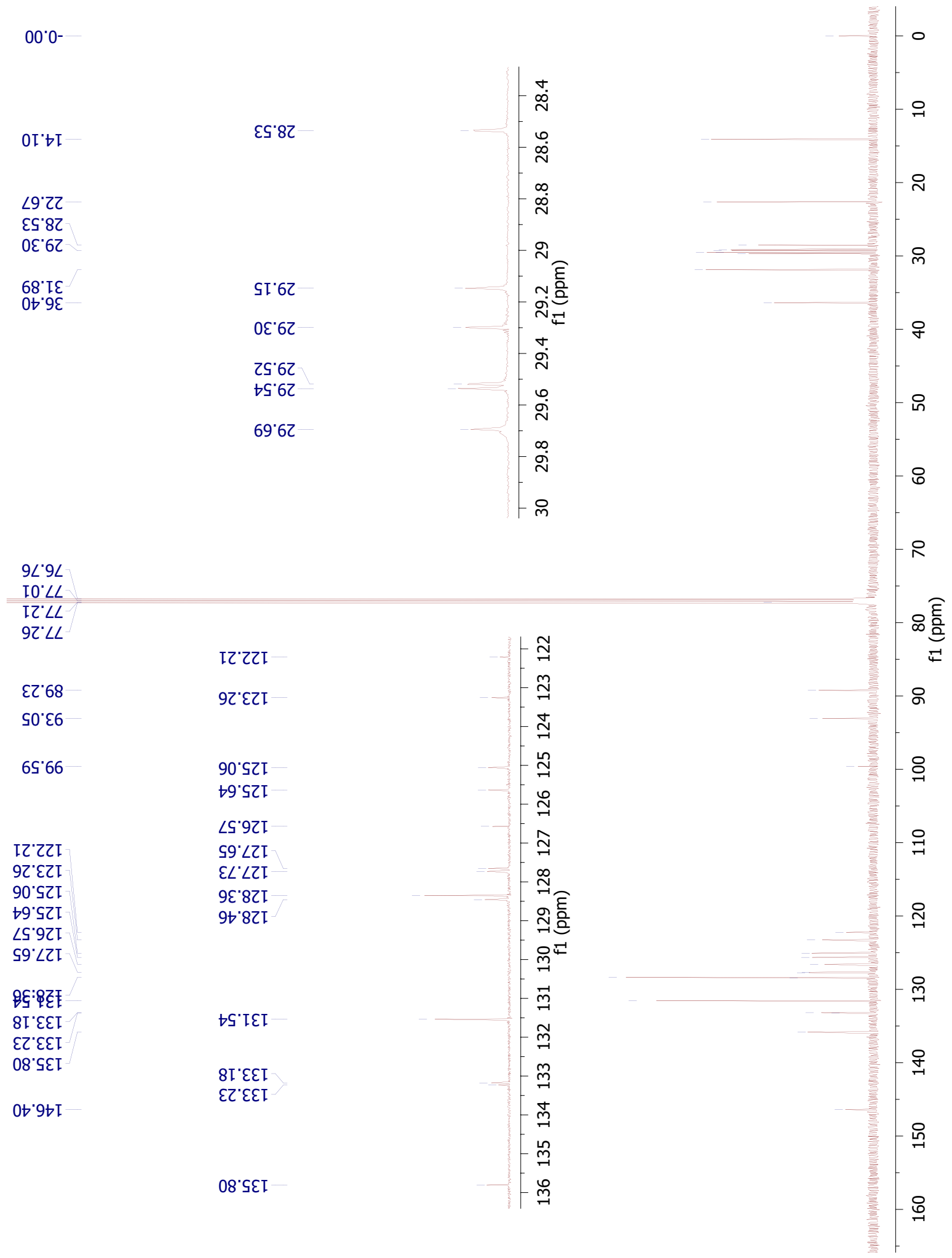

Fig. $\mathrm{S}-18:{ }^{13} \mathrm{C}$ NMR spectrum of 8 . 